\title{
Dynamic spindle reflexes and the rigidity of Parkinsonism
}

\author{
D. L. MCLELLAN ${ }^{1}$ \\ From the University Department of Neurology, Institute of Neurological Sciences, \\ Southern General Hospital, Glasgow
}

SUMMARY The effects of stimulating the reflex arc from dynamic spindle endings were examined in patients with the rigidity of Parkinsonism and in control subjects. The arc was activated phasically by a tendon tap and by electrical stimulation in 15 patients. The effect of reinforcement by Jendrassik's manoeuvre was observed. The response to phasic activation indicated central facilitation of the reflex loop in the patients with Parkinsonism, with a concurrent decrease in fusimotor drive to dynamic spindles. These abnormalities could not be correlated with the severity of the patients' rigidity, and they did not alter when the rigidity was reduced by levodopa. The effect of activating dynamic spindle endings tonically by vibration at $50 \mathrm{~Hz}$ was also examined. The reflex contraction of the biceps and triceps muscles in response to vibration was found to be increased in 24 patients with rigidity compared with 24 control subjects. Patients with severe rigidity developed a more powerful contraction in response to vibration than patients with mild rigidity. The response toon vibration was reduced by treatment with levodopa but the amount of this reduction could not be्ठ correlated with changes in the patients' rigidity.

Rigidity is a clinical term used to indicate an increased resistance of muscle to passive stretch, the resistance being approximately constant throughout the range of stretching. DennyBrown (1960) has pointed out that when a rigid limb is fully supported and the patient relaxed, the rigidity disappears and unimpaired passive movement is possible in either direction within a range of $5-10^{\circ}$. Movement of a larger range sets up a contraction in the stretched muscle which is maintained as the movement continues, showing that rigidity is a stretch reflex activated by sensory receptors in the muscle. Sensory nerve fibres from primary muscle spindle organs are known to connect monosynaptically with alpha motoneurones serving related extrafusal muscle fibres. It is therefore possible that activation of primary spindle organs initiates the reflex contraction responsible for the phenomenon of rigidity. This would imply abnormal functioning of the reflex loop and the purpose of the present

\footnotetext{
1 Present address: Department of Neurology, Division of Neurological Sciences, the London Hospital, London E1 $1 \mathrm{BB}$.
}

study was to test the operation of the dynamic spindle reflexes serving rigid muscles.

The monosynaptic reflex system may be tested in several ways (Fig. 1). A volley can be initiated in afferent nerve fibres by phasic mechanical excitation of the spindle organs (tendon tap) or by electrical stimulation of the muscle nerve, using a stimulus pulse that is subthreshold for motor fibres but above threshold for sensory ones (the ' $\mathrm{H}$ ' response: Hoffmann 1919). Such an afferent volley produces a phasic contraction in the muscle. The amplitude of the tendon jerk would be increased either if the spindle organs were excessively sensitive to mechanical stimulation because of increased fusimotor activity, or if central facilitation of the reflex arc were to occur. Central facilitation would be characterized by an increase in the amplitudes of both the tendon tap and the $\mathrm{H}$ responses. Overactivity of the fusimotor system would increase the amplitude only of the tendon tap and would therefore increase the ratio between the tendon tap and $\mathrm{H}$ responses. Alternatively, the dynamic spindles may be activated 


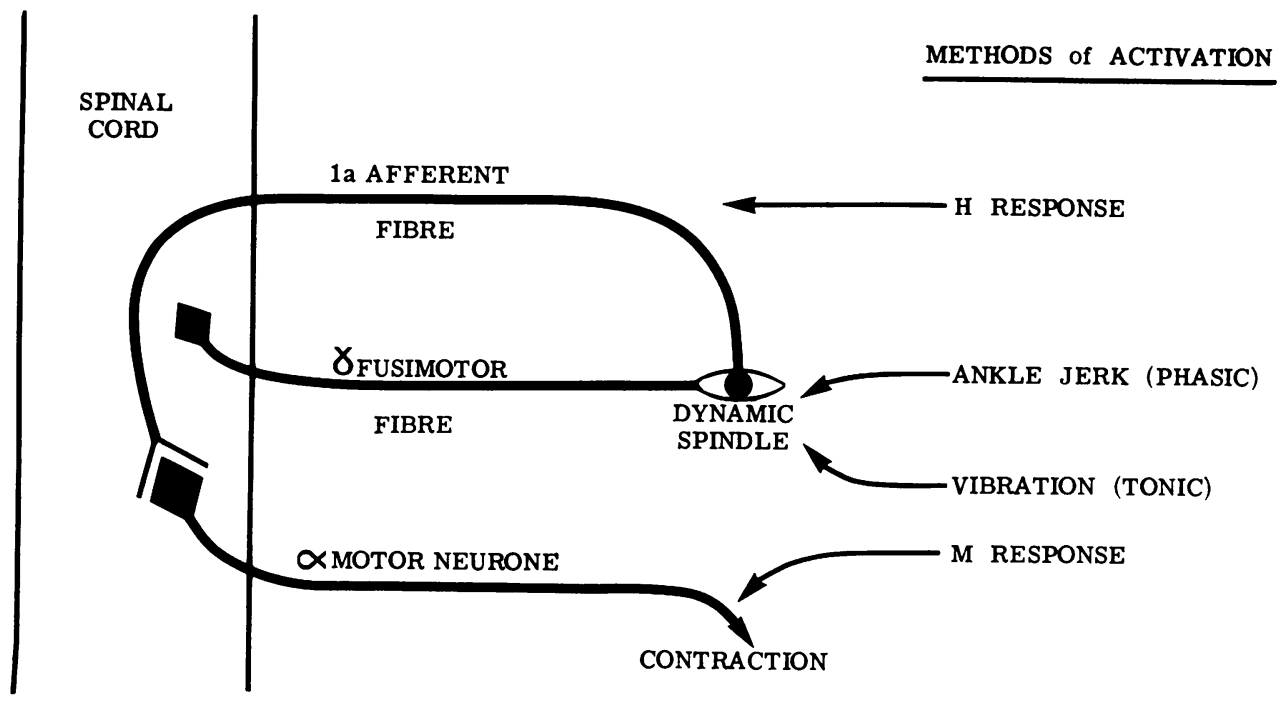

FIG. 1. Diagram of the monosynaptic reflex arc from dynamic muscle spindles, showing the point at which the arc can be activated experimentally.

tonically by vibration of the muscle or its tendon which causes a tonic contraction to build up in the muscle over the course of 10-20 seconds: this has been called the Tonic Vibration Reflex or TVR (Eklund and Hagbarth, 1966). The build-up in contraction occurs in spite of the constant level of vibration, implying that the sensitivity of the system is progressively increased during tonic activation. This change in sensitivity bears some resemblance to the phenomenon of post-tetanic potentiation (Eklund and Hagbarth, 1966). It follows that vibration of a muscle tests functions of the spindle reflex arc that are not tested by phasic stimulation.

\section{PHASIC ACTIVATION: METHOD}

SUBJECTS The effects of phasic activation of the monosynaptic arc were examined in 15 patients with rigidity before and after treatment with levodopa, and in 15 control subjects. Before treatment 12 of the 15 patients with Parkinsonism had rigidity of both legs, two had rigidity of one leg, and one had no rigidity of either leg. After treatment with levodopa 10 patients had less rigidity, improvement occurring in 17 out of the total of 30 legs. The control subjects had no clinical abnormality of the motor system. The mean age of the patients was 54.3 years (range 40-72 years) and seven were males: the mean age of the control subjects was 52.4 years (range 24-68 years) and seven were males.

PROCEDURE The subject was asked to empty his bladder and then lie on a bed in the prone position with the knee partly flexed at an angle of $30^{\circ}$, the lower leg being supported by a padded ramp as far as the ankle and the foot left free. The subject was helped to take up a comfortable position with the head turned away from the side of examination, and at least 10 minutes were spent in allowing him to become accustomed to the apparatus and the techniques before recordings were made. The laboratory was silent and without distractions. The electromyographic activity in the soleus muscle was recorded by skin-clip surface electrodes, placed $3 \mathrm{~cm}$ apart over the long axis of the muscle mid-way between the posterior and lateral borders of the calf. The position of the recording electrodes was noted, using the head of the fibula as the reference point, and subsequent recordings were made from the same site. The voltage difference between the recording electrodes was fed through a Medelec pre-amplifier to an EMG main amplifier (total gain $\times 40,000$ ) and displayed on a storage oscilloscope (Tektronix RM 564). The signals were also monitored from a loudspeaker. To elicit an $\mathrm{H}$ response stimulating electrodes were placed $3 \mathrm{~cm}$ apart, with the cathode proximal to the anode, over the medial popliteal nerve in the popliteal fossa; the position of the cathode was recorded using the head of the fibula as a reference 
point and the same site was employed in subsequent observations of each patient. A square wave electrical stimulus lasting $1 \mathrm{msec}$ was delivered from a Disa Ministim stimulator that simultaneously triggered the oscilloscope. The voltage of the stimulus was gradually increased until an $\mathbf{H}$ response of maximum amplitude was evoked, whether or not an additional $M$ response also occurred. The amplitude of the $\mathbf{H}$ response obtained in this way was more consistent than the amplitude of near-threshold $\mathrm{H}$ responses, a conclusion reached also by Olsen and Diamantopoulos (1967).

The ankle jerk (AJ) was elicited by striking the achilles tendon with a manually operated hammer, the head of which contained an electrical switch that triggered the oscilloscope as the tendon was struck. The blow was directed at a marked point at which adjoining muscle became palpable; the direction of the blow was at right angles to the skin and the force of the blow was chosen so that a maximal response was elicited, avoiding inhibition of the response by pain or apprehension in the subject. The tendon was struck 20 times at intervals of approximately 30 seconds, the patient being instructed to perform Jendrassik's manoeuvre immediately before alternate taps. The presence of voluntary activity in the muscle was monitored through the loudspeaker and none occurred during Jendrassik's manoeuvre. The peak to peak amplitude of the electromyographic $\mathrm{AJ}$ response was recorded on each

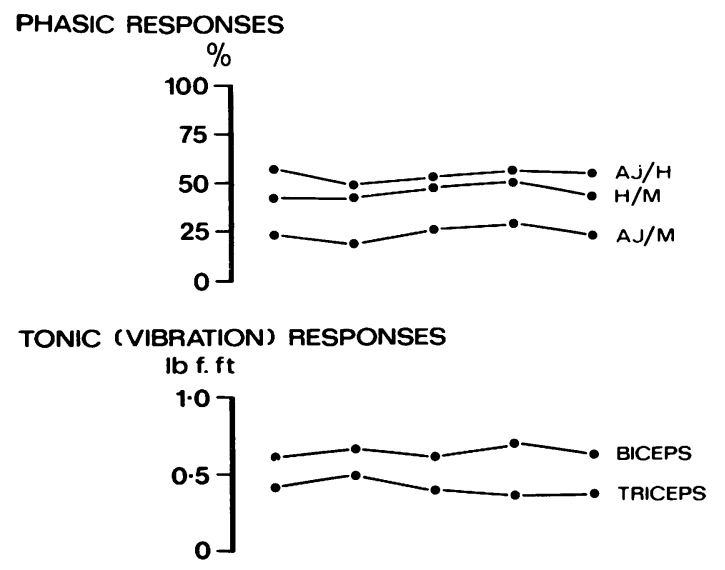

FIG. 2. Variability of phasic and tonic responses in the same subject on different occasions: the five sets of readings were obtained on different days over a period of three weeks. AJ, ankle jerk; $\mathrm{H}, \mathrm{H}$ response; $\mathrm{M}$, maximal twitch response of the soleus-gastrocnemius muscle; for further explanation see text. occasion. The maximal $\mathrm{H}$ response was also obtained $\stackrel{\overline{\mathrm{o}}}{\mathrm{c}}$ 20 times at intervals of approximately 30 seconds, $\underset{\mathbb{D}}{Z}$ with facilitation of alternate responses by Jendrassik's manoeuvre, and the peak to peak amplitude of each of $\mathrm{H}$ response was recorded. The maximal $\mathrm{M}$ response $\underset{0}{\widehat{C}}$ was obtained by using a supramaximal electrical 0 stimulus-that is, of a voltage $50 \%$ greater than that necessary to produce a maximal $M$ response. The peak to peak amplitude of the maximal $M$ response $\stackrel{\overrightarrow{0}}{\overrightarrow{7}}$ was recorded. The position of the stimulating elec-. trodes and of the recording electrodes was unchanged $\underset{\vec{P}}{\vec{P}}$ throughout the period of observation.

Studies of normal subjects and of patients showed $\frac{}{0}$ that these techniques enabled amplitude ratios of $\frac{\bar{F}}{\frac{5}{1}}$ $\mathrm{AJ} / \mathrm{M}, \mathrm{H} / \mathrm{M}$, and $\mathrm{AJ} / \mathrm{H}$ to be calculated that were $\overrightarrow{\mathbb{D}}$ consistently reproducible for any one subject $\frac{\circ}{\nu}$ examined on different occasions (Fig. 2).

\section{PHASIC ACTIVATION: RESULTS}

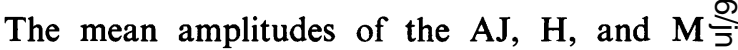
responses and of the reinforced AJ (AJR) and reinforced $H$ responses (HR) were recorded. The $\omega$ ratios between them were expressed as percent $\omega$ ages and are shown in the Table.

\section{TABLE}

RATIOS BETWEEN MEAN AMPLITUDES OF ANKLE JERK, REINFORCED ANKLE JERK, H RESPONSE, REINFORCED H RESPONSE, AND M RESPONSE IN CONTROL SUBJECTS AND IN PATIENTS BEFORE AND AFTER TREATMENT WITH LEVODOPA

\begin{tabular}{lccc}
\hline Ratio $(\%)$ & $\begin{array}{c}\text { Control } \\
\text { subjects }\end{array}$ & \multicolumn{2}{c}{ Patients with Parkinsonism } \\
\cline { 3 - 4 } & & Untrcated & $\begin{array}{c}\text { After } \\
\text { levodopa }\end{array}$ \\
\hline AJR/AJ & 157.3 & $160 \cdot 1$ & 160.6 \\
HR/H & 123.1 & 121.9 & 123.1 \\
AJ/M & 12.7 & 16.6 & 19.2 \\
H/M & 22.4 & $36.8^{*}$ & 42.0 \\
AJ/H & 70.6 & $45 \cdot 1^{*}$ & 44.9 \\
\hline
\end{tabular}

* Statistically significant difference between untreated patients and

$A J=$ ankle jerk. $H=H$ response. $H R=$ reinforced $H$ response. $M=$ $M$ response.

EFFECT OF REINFORCEMENT The effects of re- $D$ inforcement of $\mathrm{AJ}$ and $\mathrm{H}$ responses by the Jendrassik manoeuvre were approximately the $N$ same in the patients as in the controls. The $\mathrm{H}$ response was facilitated to $120 \%$ of its previous amplitude. Although the effect upon the AJ was 
proportionally greater, it is clear that facilitation did not depend upon changes in the mechanical sensitivity of spindle endings-in other words, Jendrassik's manoeuvre increased the number of motoneurones activated by an incoming volley. No significant change occurred with levodopa.

H/M RATIO The mean $\mathrm{H} / \mathrm{M}$ ratio was higher in untreated patients than in the controls $(\mathrm{P}<0.02)$. This indicates central facilitation of a phasic incoming volley. A slight increase in this ratio occurred during treatment but was not statistically significant. The $H / M$ ratio in limbs with marked rigidity was the same as that in limbs with mild rigidity. There was no relationship between the $H / M$ ratio and the severity of rigidity.

AJ/M RATIO The mean $\mathrm{AJ} / \mathrm{M}$ ratio, which corresponds with the clinical tendon jerk, was slightly greater in patients than in controls but the difference was not statistically significant; a small increase in the ratio occurred during treatment.

AJ/H RATIO As would be expected from the $\mathrm{H} / \mathrm{M}$ and $\mathrm{AJ} / \mathrm{M}$ ratios, the patients with Parkinsonism had a lower mean $\mathrm{AJ} / \mathrm{H}$ ratio than the controls $(P<0.02)$ but no significant change occurred during treatment. Patients with mild rigidity and patients with severe rigidity had similar $\mathrm{AJ} / \mathrm{H}$ ratios. The finding of a normal ankle jerk in the presence of an increased $\mathrm{H}$ response indicates that, in the patients studied, the sensitivity of the dynamic spindle organs to mechanical stimulation was reduced-in other words, there was diminished activity in fusimotor fibres to dynamic spindles. The relevance of these findings to the patients' rigidity and to previous investigations is considered in the discussion.

\section{TONIC ACTIVATION: METHOD}

SUBJECTS The force developed by the reflex contraction of the biceps and triceps muscles in response to vibration was measured in 24 patients with Parkinsonism. The patients were selected because they had rigidity and only one arm among the 24 patients was regarded as having clinically normal tone. Fourteen of the patients were reassessed after treatment with levodopa, when rigidity was found to have been reduced in 10 (15 arms). Twenty-four control subjects who had no abnormality of the motor system were also assessed. The mean age of the patients was 55.4 years (range $35-72$ years) and 14 were males; the mean age of the control subjects was 55.5 years (range 24-81 years) and 14 were males. The mean height of the control subjects was $166.9 \mathrm{~cm}$ and of the patients was $165.6 \mathrm{~cm}$; the mean body weight was $66 \cdot 1 \mathrm{~kg}$ and $65 \cdot 3 \mathrm{~kg}$ respectively.

PROCEDURE The subject was seated comfortably in a chair while each arm in turn was clamped in an adjustable load measuring splint, the angle of the elbow being fixed at $30^{\circ}$ from full extension. The forearm was in pronation and the wrist and fingers held straight. The limb was firmly attached to the splint at points $10 \mathrm{~cm}$ above the elbow, at the wrist, and at the middle phalanges of the fingers which were held together in an adjustable metal loop. The medial condyle of the humerus rested upon a firm pad within the splint. The patient was allowed to become accustomed to the apparatus. Flexion and extension of the elbow was possible between readings. The patient was asked to look straight ahead with the eyes closed; this was found to be more satisfactory than blindfolding as it did not alarm elderly subjects. The biceps and triceps muscles were vibrated in turn by a Pifco vibrator operating at maximum amplitude at a rate of $50 \mathrm{~Hz}$ and applied to the distal portion of the muscle belly approximately $5 \mathrm{~cm}$ above the elbow joint. The pressure of application was adjusted to produce a maximum contraction in the muscle: too light a pressure would activate too few spindles but too great a pressure reduced the excursion of the vibrator, thereby reducing its effect (Eklund and Hagbarth, 1966). The torque generated by the vibrated muscle was measured by a transducer consisting of a steel strip attached to the splint, with four metal foil strain gauges mounted in a full bridge configuration. Vibration was continued until a steady state was reached, usually at $20-30$ seconds. Occasionally the torque level would drop suddenly from this plateau as the patient re-adjusted his arm, but the plateau was repeatedly regained as vibration continued. Repeated measurements of the torque using this technique confirmed that results could be obtained that were consistently reproducible for any one subject examined on different occasions (Fig. 2).

\section{TONIC ACTIVATION: RESULTS}

The torque induced by vibration of the patients' muscles was greater than that produced in normal subjects (Fig. 3), statistical analysis by Student's $t$ test giving $\mathrm{P}<0.001$ for the biceps and $\mathrm{P}<0.01$ for 


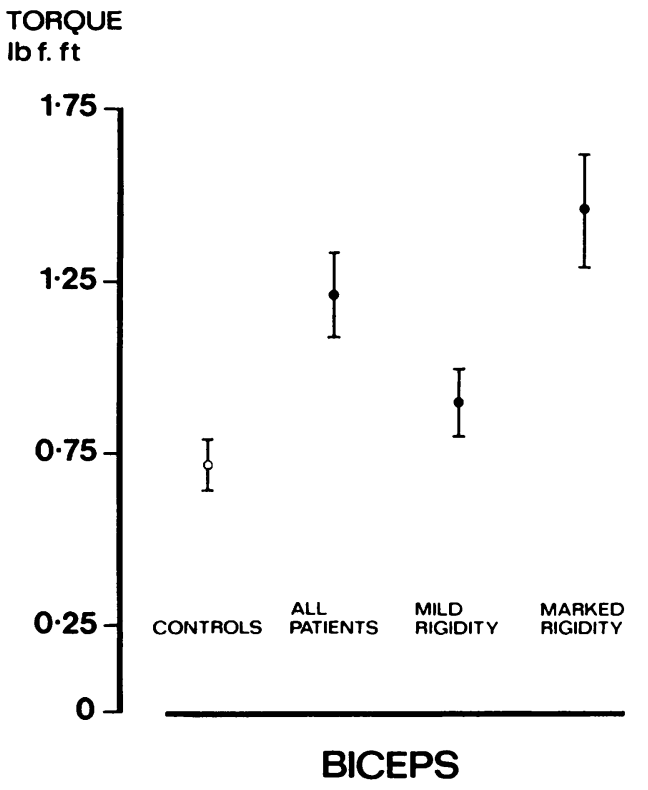

(a)

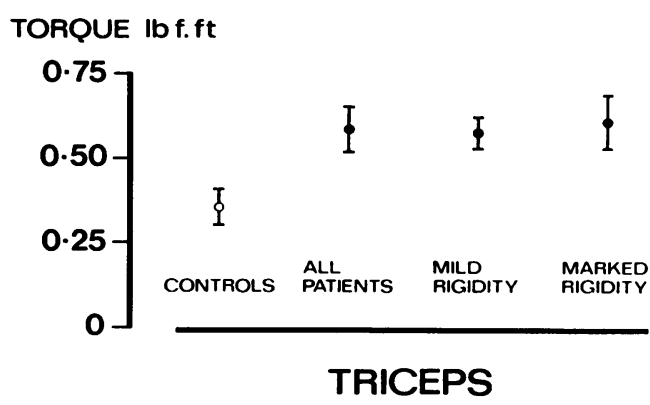

(b)

FIG. 3. Torques developed by vibration of the biceps (a) and triceps (b) muscles of 24 patients with Parkinsonism and in 24 subjects with normal muscle tone. Torque is indicated on the vertical scale. Mean torque values $( \pm$ one $S E)$ are shown.

the triceps muscles. The degree of stretch of the triceps muscle during these observations would tend to reduce its TVR relative to that of the biceps muscle, so that no conclusions can be drawn about possible differential responses to vibration in flexor and extensor muscles. The patients with marked rigidity developed greater torques than those with mild rigidity $(\mathrm{P}<0.01$ for the biceps muscle). After

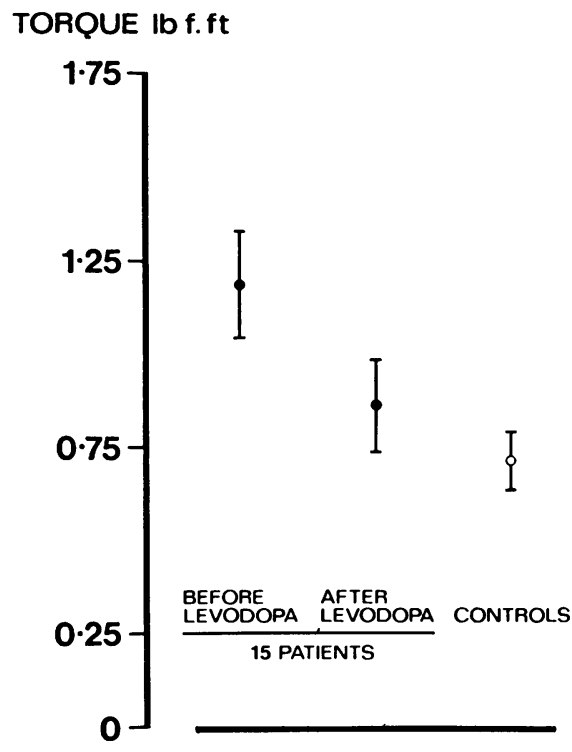

BICEPS

(a)

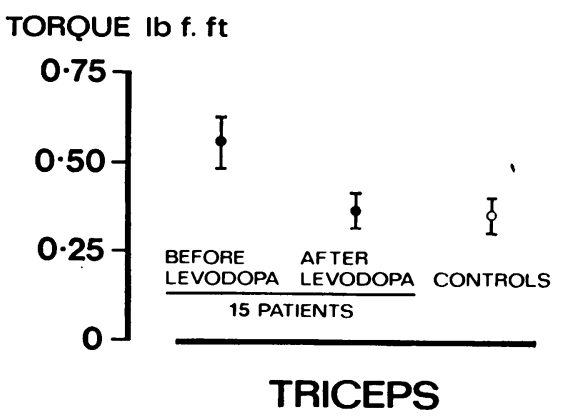

(b)

FIG. 4. Torques developed by vibration of the biceps (a) and triceps (b) muscles of 15 patients with Parkinsonism before and after treatment with levodopa, compared with that obtained in 24 control subjects. Torque is indicated on the vertical scale. Mean torque values ( \pm one $S E$ ) are shown.

treatment with levodopa, the torque generated by $\bigcirc$ vibration was reduced but did not fall to the control level (Fig. 4). Patients whose rigidity improved as a result of treatment did not show a greater reduction in torque than patients whose rigidity was clinically unchanged by treatment.

Comparison of the mean height and weight of the $\mathrm{C}$ patients and control subjects showed no marked 
difference between the groups, nor was there a difference in body build between patients with severe rigidity and those with mild rigidity. It is, therefore, reasonable to conclude that the greater response to vibration in patients with rigidity was related to their rigidity and not to anatomical differences between the arms of the various subject groups.

In patients whose rigidity was clinically unchanged during treatment, the response to vibration was nevertheless reduced by levodopa and the effect of levodopa upon the TVR could not be correlated with its effect upon rigidity.

\section{DISCUSSION}

There is no doubt that rigidity is a stretch reflex initiated by receptors in the passively stretched muscle but electromyographic studies of stretch reflexes in patients with Parkinsonism have thrown little light upon the functional abnormalities underlying rigidity. This has been partly because of the difficulties of clinical measurement and partly because the pattern of disability varies considerably from patient to patient, even in the untreated state. The recent introduction of levodopa has further complicated the neurophysiological investigation of Parkinsonism because levodopa not only alters the signs of the disease but may have other reflex effects not necessarily related to its therapeutic action.

Controversy about the peripheral function of stretch reflexes in Parkinsonism has centred upon the mechanical sensitivity of the dynamic muscle spindles, which is taken to be an index of gamma motoneurone activity. The muscle 'silent period', produced by electrical stimulation of a mixed peripheral nerve, has been shown by several authors to be normal in patients with the rigidity of Parkinsonism. Although the silent period may, in certain circumstances, reflect muscle spindle activation, the period of silence resulting from a supramaximal electrical stimulus results directly from the stimulation of afferent nerve fibres and therefore gives no indication of spindle activity in the muscle (McLellan, 1973). Studies of the electrically-produced silent period are, therefore, of limited value, and possibly of no value, in the investigation of spindle function in man.

Certain abnormalities of the monosynaptic reflex in Parkinsonism are well documented but are of limited value because pooled data from heterogeneous groups of patients have been presented, without an attempt to correlate the findings with clinical data from individual patients. The refractory period and subsequent recovery of the $\mathrm{H}$ reflex is abnormal in Parkinsonism but the physiological significance of this phenomenon is obscure (Ioku, Ribera, Cooper, and Matsouka, 1965; Olsen and Diamantopoulos, 1967; Yap, 1967).

There is disagreement about the effect of Jendrassik's manoeuvre in Parkinsonism, a subject briefly reviewed by Calne (1970). The present findings agree with those of Gassel and Diamantopoulos (1964) that both maximal AJ and maximal $\mathrm{H}$ responses are facilitated by Jendrassik's manoeuvre, the degree of facilitation being no different from that obtained in normal subjects. Care was taken in the present study to obtain consistently maximal $\mathrm{H}$ responses before Jendrassik's manoeuvre so that their subsequent facilitation must have occurred within the spinal cord. It is reasonable to assume that the total motoneurone pool available to the monosynaptic arc is limited. A small incoming volley would be more readily reinforced than one that was large enough to activate most of the available motoneurone pool. As the AJ response was generally smaller than the $\mathbf{H}$ response, it is not surprising that Jendrassik's manoeuvre gave rise to a proportionally greater facilitation of the AJ response. It is not necessary to postulate that Jendrassik's manoeuvre increases fusimotor drive concurrently with the increase in central facilitation, though it is true that such a mechanism cannot be excluded by the present observations.

The way in which the monosynaptic loop handles phasic reflexes may not be directly relevant to rigidity; such phasic stimuli are rarely experienced in the normal course of events. There is no characteristic clinical abnormality of tendon reflexes in Parkinsonism which may be increased, normal, or diminished. The present study has confirmed that there is no significant difference between the mean $\mathrm{AJ} / \mathrm{M}$ ratios of patients and control subjects. There was, however, an increase in the $\mathrm{H} / \mathrm{M}$ ratio compared with normal subjects suggesting an increase in control sensitivity or 'gain' of the monosynaptic loop to an incoming volley from primary spindle receptors. The degree of 
enhancement did not correlate with the severity of rigidity and it increased further during treatment with levodopa despite a reduction in rigidity.

The discrepancy between the $\mathrm{AJ} / \mathrm{M}$ and $\mathrm{H} / \mathrm{M}$ findings is reflected by the $\mathrm{AJ} / \mathrm{M}$ ratio, that indicates a significant decrease in dynamic fusimotor activity in the patients with Parkinsonism. The $\mathrm{AJ} / \mathrm{H}$ ratio could not be correlated with the severity of rigidity. Procaine injected into a rigid muscle reduces its tone, presumably by its preferential action on fusimotor fibres; this has been interpreted as evidence of increased fusimotor drive in rigidity (Russell, 1960). The present findings do not support such a conclusion but they are consistent with the hypothesis that by further reducing fusimotor drive, procaine reduces the sensory input available for facilitation within the spinal cord. It is of interest that the net effect of reduced fusimotor drive and increased central facilitation was to maintain a virtually normal tendon tap response. It is possible that this balance reflects a reflex adjustment by the nervous system to an abnormality of one of these mechanisms. The possibility of a relationship between these findings and the patients' bradykinesia is being investigated.

Previous comparisons of the $\mathrm{H}$ reflex amplitude in normal and rigid subjects have failed to show any abnormality (Angel and Hofmann, 1963; Dietrichson, 1971) but the AJ/H response ratio was found to be increased by Dietrichson
(1971) despite normal $\mathrm{H}$ responses and the fact that the mechanical threshold of rigid subjects was normal. In the latter study, the control subjects were considerably younger than the patients and the measured maximal $\mathrm{AJ}$ and $\mathrm{H}$ amplitudes were given, rather than the ratio between the mean of a series of $\mathrm{H}$ responses and the $\mathrm{M}$ response. Differences in skin conductivity and subcutaneous tissue thickness would therefore be added to the already substantial difficulties inherent in comparing one set of subjects with another. However carefully selected, a group of patients with Parkinsonism is inevitably heterogeneous, so that quantitative comparisons between such a group and normal subjects may not be so secure as they at first appear. However, the results of the comparison become more significant if a positive correlation can be found with a particular clinical feature in the patients, and changes in reflex activity can be shown to accompany clinical improvement during treat- $\omega$ ment. From the present study and from others $\omega$ quoted, it is reasonable to conclude that rigiditi $\omega$ is not consistently related to any disorder of $\vec{N}$ phasic monosynaptic reflexes.

The responses to vibration reported here suggest that tonic activation of the dyname spindles has an enhanced effect in the rigidity Parkinsonism and that the degree to which $\overline{9}$ tonic input is enhanced (in contrast to a phasîc input) reflects the severity of the patient's rigidity. Although all the patients whose rigidity was improved by levodopa showed a reduction
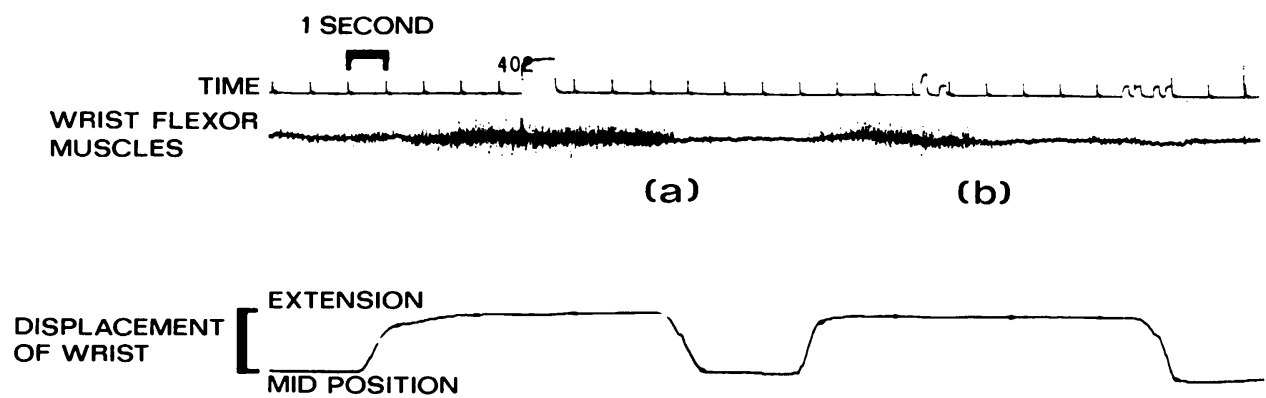

FIG. 5. Supraspinal factors in rigidity: the effect of passive stretch and conscious relaxation upon a rigid muscle. Upper trace: surface electromyogram of wrist flexor muscles; lower trace: displacement of the wrist. The patient is asked to relax. Passive stretching of the muscle then induces a reflex contraction that disappears either when the stretch is removed (a) or when the patient makes a new conscious effort to relax (b). 
in their TVR, a reduction in the TVR was not always accompanied by a reduction in clinical rigidity so that the precise relationship between rigidity, the TVR, and the effect of levodopa requires further investigation. A previous study of the TVR in Parkinsonism suggested that the patients had a normal response to vibration, in contrast with patients with spasticity whose response was reduced (Lance, de Gail, and Neilson, 1966). The present study was conducted with a large number of subjects and the Parkinsonism and control groups were of comparable age, sex, and body build.

During normal daily activity most skeletal muscles are active for most of the time and this activity involves continual discharges from spindle endings. Clinical evaluation of patients in normal circumstances would therefore test the activity of the stretch reflex against this background. After a patient has relaxed on a couch for several minutes, however, rigidity may be greatly reduced and this could reflect the absence of conditioning discharges from the muscle spindles during the preceding period of relaxation. In addition, and of probably more importance, is the fact that supraspinal factors have a considerable influence both upon the TVR and upon rigidity-for example, the resistance of rigid muscles to maintained stretch is characteristically abolished during conscious relaxation of the limb by the patient himself (Fig. 5), while the TVR is easily abolished by the patient who makes a conscious effort to suppress it. The similarity between the TVR and post-tetanic potentiation has already been noted (Eklund and Hagbarth, 1966) and it is known that segmental reflex activity can be controlled by supraspinal influences (Lundberg, 1967).

Finally, it should be emphasized that the techniques used in this study provide no information about the control and behaviour of static muscle spindles. When a rigid muscle is maintained in a fixed position of stretch a constant contraction is induced in the muscle, which often continues until the stretch is removed (Fig. 5). Clearly such a response cannot be maintained by an input from dynamic spindles which are velocity-sensitive rather than length-sensitive (Matthews, 1971). It is likely that reflexes in- volving both varieties of muscle spindle are disturbed in rigidity.

I wish to thank the subjects for their cooperation and Professor J. A. Simpson for his encouragement and advice. I am also grateful to the other consultant neurologists at the Institute of Neurological Sciences for the opportunity to study patients under their care; to Mr. Harry Leigh for valuable technical assistance, and to Roche Products Limited for supplies of levodopa.

\section{REFERENCES}

Angel, R. W., and Hofmann, W. W. (1963). The H reflex in normal, spastic, and rigid subjects. Archives of Neurology, 8, 591-596.

Calne, D. B. (1970). Rigidity. In Parkinsonism: Physiology, Pharmacology and Treatment, pp. 40-41. Arnold: London.

Denny-Brown, D. (1960). Diseases of the basal ganglia. Their relation to disorders of movement. 2. Lancet, 2, 1155-1162.

Dietrichson, P. (1970). Phasic ankle reflex in spasticity and Parkinsonian rigidity. Acta Neurologica Scandinavica, 47, 22-51.

Eklund, G., and Hagbarth, K. E. (1966). Normal variability of tonic vibration reflexes in man. Experimental Neurology, 16, 80-92.

Gassel, M. M., and Diamantopoulos, E. (1964). The Jendrassik maneuver. $I$. The pattern of reinforcement of monosynaptic reflexes in normal subjects and in patients with spasticity or rigidity. Neurology (Minneap.), 14, 555560.

Hoffmann, P. (1920). Demonstration eines Hemmungsreflexes im menschlichen Rückenmark. Zeitschrift für Biologie, 70, 515-524.

Ioku, M., Ribera, V. A., Cooper, I. S., and Matsouka, S. (1965). Parkinsonism: electromyographic studies of monosynaptic reflex. Science, 150, 1472-1475.

Lance, J. W., de Gail, P., and Neilson, P. D. (1966). Tonic and phasic spinal cord mechanisms in man. Journal of Neurology, Neurosurgery, and Psychiatry, 29, 535-544.

Lundberg, A. (1967). The supraspinal control of transmission in spinal reflex pathways. Electroencephalography and Clinical Neurophysiology. Suppl. 25, 35-46.

McLellan, D. L. (1973). The electromyographic silent period produced by supramaximal electrical stimulation in normal man. Journal of Neurology, Neurosurgery, and Psychiatry, 36, 334-341.

Matthews, P. B. C. (1971). Recent advances in the understanding of the muscle spindle. In The Scientific Basis of Medicine Annual Reviews, 1971, pp. 99-128. Edited by I. Gilliland and J. Francis. Athlone Press: London.

Olsen, P. Z., and Diamantopoulos, E. (1967). Excitability of spinal motor neurones in normal subjects and in patients with spasticity, Parkinsonism rigidity, and cerebellar hypotonia. Journal of Neurology, Neurosurgery, and Psychiatry, 30, 325-331.

Rushworth, G. (1960). Spasticity and rigidity: an experimental study and review. Journal of Neurology, Neurosurgery, and Psychiatry, 23, 99-118.

Yap, C. B. (1967). Spinal segmental and long-loop reflexes on spinal motoneurone excitability in spasticity and rigidity. Brain, 90, 887-896. 